\title{
DENSE, PARSEC-SCALE CLUMPS NEAR THE GREAT ANNIHILATOR
}

\author{
Edmund Hodges-Kluck ${ }^{1}$, Marc W. Pound ${ }^{1}$, Andrew I. Harris ${ }^{1}$, James W. Lamb ${ }^{2}$, and Mark Hodges ${ }^{2}$ \\ ${ }^{1}$ Department of Astronomy, University of Maryland, College Park, MD 20742-2421, USA \\ ${ }^{2}$ Owens Valley Radio Observatory, California Institute of Technology, Big Pine, CA 93513, USA \\ Received 2008 October 6; accepted 2009 February 17; published 2009 April 24
}

\begin{abstract}
We report on Combined Array for Research in Millimeter-Wave Astronomy and James Clerk Maxwell Telescope observations toward the Einstein source 1E 1740.7-2942, a low-mass X-ray binary commonly known as the "Great Annihilator." The Great Annihilator is known to be near a small, bright molecular cloud in a region largely devoid of emission in ${ }^{12} \mathrm{CO}$ surveys of the Galactic center. This region is of interest because it is interior to the dust lanes which may be the shock zones where atomic gas from the HI nuclear disk is converted into molecular gas. We find that the region is populated with a large number of dense $\left(n \sim 10^{5} \mathrm{~cm}^{-3}\right)$ regions of excited gas with small filling factors. The gas appears to have turbulent support and may be the result of sprays of material from collisions in the shock zone. We estimate that $\sim(1-3) \times 10^{5} M_{\odot}$ of shocked gas resides in our $r \sim 3^{\prime}$, $\Delta v_{\mathrm{LSR}}=100 \mathrm{~km} \mathrm{~s}^{-1}$ field. If this gas has recently shocked and is interior to the inner Lindblad resonance of the dominant bar, it is in transit to the $x_{2}$ disk, suggesting that a significant amount of mass may be transported to the disk by a low filling factor population of molecular clouds with low surface brightness in larger surveys.
\end{abstract}

Key words: Galaxy: center - ISM: clouds

\section{INTRODUCTION}

In spite of the difficulties in determining the structure and dynamics of the inner $400 \mathrm{pc}$ of the Galaxy, remarkable progress has been made by comparing surveys in different tracers of molecular gas. These surveys have revealed the average properties of the gas (Bania 1977; Liszt \& Burton 1978; Bally et al. 1987; Stark et al. 1988; Bitran et al. 1997; Oka et al. 1998; Tsuboi et al. 1999; Martin et al. 2004). The gas is excited, dense, and exists in conditions unique in the Galaxy, including high external pressures and strong magnetic fields. The most prominent line emission comes from the various rotational energy levels of ${ }^{12} \mathrm{CO}$, and surveys exist from $J=1-0$ to $J=7-6$. It is evident from these degree-scale results that the molecular medium is not well described by its average properties, but over the past few decades a convincing picture of the Galactic center has emerged. At least one stellar bar crosses the Galaxy, and the $x_{1}$ and $x_{2}$ orbit families resulting from its potential contain atomic and molecular gas respectively (Binney et al. 1991). The molecular gas on the $x_{2}$ orbits is largely found in a "Galactic center ring" (GCR) and is both dense and turbulent (Linke et al. 1981; Fux 1999). Because gas on the GCR is forming stars (e.g., Qin et al. 2008), it is unlikely that the molecular medium inside the $x_{1}$ disk is primordial, and therefore some mechanism must exist for converting atomic gas to molecular gas. The region in between the atomic disk and the GCR, the site of the bar's inner Lindblad resonance (ILR), is where the transition occurs, and is thought to contain two dust lanes in small spiral arms where molecule-forming shocks and sprays occur (Rodriguez-Fernandez et al. 2006). The GCR and surrounding molecular gas near these dust lanes are collectively known as the "central molecular zone" (CMZ). The most obvious failure of this model is that it has not yet explained the asymmetric distribution of gas around the dynamical center of the Galaxy, Sgr A*, although Rodriguez-Fernandez \& Combes (2008) argue that the GCR may be accreting from only one side.

This simple picture is remarkable considering the observed complexity of the region. However, it necessarily overlooks details critical to a complete understanding. In particular, we are interested in the specific nature of the process converting atomic gas to molecular gas. That the dust lanes are the site of moleculeforming shocks is likely given the absence of star formation, and studies in line ratios tracing shock chemistry indicate that the lanes contain shocked gas (Rodriguez-Fernandez et al. 2006), but the shock environment has yet to be explored in detail. Preand postshock environments appear as relative voids in $(l, v)$ diagrams; the emptiness of the region varies with the choice of spectral line, but even in ${ }^{12} \mathrm{CO}(1-0)$, it is clear that there are empty regions within the CMZ, inside what Binney et al. (1991) saw as the "CO parallelogram" and earlier studies identified as an "expanding molecular ring." This boundary is likely several structures including the molecule-forming shock zones (Rodriguez-Fernandez \& Combes 2008), and since shocked gas must fall farther into the Galactic potential, the relative paucity of emission interior to the parallelogram is interesting. There are several possible explanations for the voids including (1) the shocked gas primarily joins the $x_{2}$ disk at certain regions (e.g., the $l=+1.3$ cloud), (2) the filling factor of shocked gas in these regions is too low to be seen by large-scale surveys (along the lines of the suggestion of Oka et al. 1998), (3) the newly formed molecular bundles are extremely diffuse or not emissive, or a combination of the above. We expect that studying the postshock side of the dust lanes will provide information regarding the state in which the gas arrives on the $x_{2}$ disk, and may also be a useful contrast to molecular cloud formation in dust lane shocks in the disk.

\subsection{The Great Annihilator}

Motivated by published data (Bally \& Leventhal 1991), we chose to observe the area near a bright molecular cloud near the far side dust lane at $l=-0.9, b=-0.1$, and $v_{\mathrm{LSR}}=-140$ $\mathrm{km} \mathrm{s}^{-1}$ which lies in such a void in ${ }^{12} \mathrm{CO}(1-0)$ and HCN $(1-0)$ surveys (Bally et al. 1987; Lee 1996). Historically, this cloud has been of interest because of its coincidence on the sky with the low mass X-ray binary (LMXB) candidate known as the "Great Annihilator" (Einstein source 1E 1740.7-2942). The Great Annihilator is a bright X-ray and $\gamma$-ray source and 
was originally thought to be a black hole accreting directly from a host molecular cloud which, in turn, was brightened by the association (Bally \& Leventhal 1991; Mirabel et al. 1991). The original hypothesis of Bondi-Hoyle accretion from the interstellar medium is almost certainly incorrect given the present understanding of the system as a LMXB (Main et al. 1999), but as Weidenspointner et al. (2008) proposed a correlation in spatial distribution of LMXB systems and diffuse $\gamma$-ray emission in the Galaxy, the strong and narrow annihilation line still makes the possibility of physical association intriguing. Historically, the two strongest pieces of evidence for such an association were the stated small probability $(\sim 5 \%)$ of a chance association with a bright molecular cloud in the region (Bally \& Leventhal 1991) and the apparent offset in $\mathrm{HCO}^{+}(1-0)$ emission from the jets seen with the VLA (Phillips \& Lazio 1995). This offset was attributed to the high levels of ionization which would destroy the $\mathrm{HCO}^{+}$close to the black hole, and Lepp \& Dalgarno (1996) suggested that HCN may be able to survive in more highly ionized gas, proposing future observations.

Our interest in the gas dynamics of the inner Galaxy led us to consider the Great Annihilator region as a target for a pathfinding observation for two reasons: (1) the bright cloud is in a "postshock void" region and (2) it has a well documented brightness and spectrum in several molecular lines (Bally \& Leventhal 1991; Mirabel et al. 1991; Phillips \& Lazio 1995). This cloud is thus a reliable pathfinder target for a larger map in the region near the shock zones. It is desirable to have a known bright target included in a wide field both for orientation and determining the suitability of a given instrument to our science goals. Additionally, even supposing the LMXB is responsible for the brightness of the $v_{\mathrm{LSR}}=-140 \mathrm{~km} \mathrm{~s}^{-1}$ cloud, it is not responsible for the presence of the dense gas. The detection of one bright cloud, therefore, suggests the presence of additional molecular gas.

\subsection{Typical Voids}

However, it is imperative to consider whether the small region near the Great Annihilator is typical of the void regions we wish to study; determining whether a relative void is "typical" requires choosing a scale. We do this by considering both an observational strategy and our broader science goals. We have reasons to expect the detection of emission in these voids based on the publically available Bally et al. (1987) ${ }^{12} \mathrm{CO}(1-0)$ data. In the published $l-v$ maps of these data, which have been integrated over several $1^{\prime}$ grid points in latitude, the relative voids appear empty, but an examination of the $l-v$ maps for each slice in latitude shows faint molecular emission in parts of these regions. An absolute void across all latitude slices is probably actually empty, but when integrating across several slices, a faint structure with little extent in latitude will become fainter relative to extended structures nearby. For example, in the Bally et al. (1987) data between $-0.11<b<-0.076$, there appears to be a bridge of emission between the $\mathrm{CO}$ parallelogram shock lanes and the $x_{2}$ orbits at $l=-0.3, v_{\mathrm{LSR}}=120 \mathrm{~km} \mathrm{~s}^{-1}$. This emission occurs in a region we would expect to be mostly empty according to bar parameters such as those in RodriguezFernandez \& Combes (2008), but may be related to the highly excited bundles of gas seen in earlier Jenkins \& Binney (1994) work. Closer to the Galactic plane, this bridge does not appear, whereas the surrounding $x_{2}$ disk and shock lane structures persist at the same longitudes; the region appears in the integrated map only as a void. Interior to the CO parallelogram, a "typical" region for detailed study ought to be large enough to probe both the apparently empty regions as well as some of the faint emission.

For a detailed interferometric study of the region, the scale should clearly be much larger than an individual molecular cloud's size, and we postulate that the projected radius of the cloud associated with the Great Annihilator is typical. Assuming interaction with the LMXB as well as some intrinsic brightness, this measured radius is only influenced by the presence of the LMXB to the extent that the LMXB is responsible for the brightness of the cloud, i.e. the ratio between excitation produced by the Great Annihilator's particle emission and that produced by other means. Based on the evidence for a welldefined spectral peak at $v_{\mathrm{LSR}}=-140 \mathrm{~km} \mathrm{~s}^{-1}$ centered spatially at some distance from the LMXB, as well as the physical arguments for how far the positrons could travel (Phillips \& Lazio 1995), it seems likely that the measured radius is close to the physical one. As discussed in Section 1.1, the LMXB may not be physically associated with the cloud at all. The field of view must be large enough to isolate individual molecular clouds of this size (or small structures), but small enough that good $u-v$ coverage and sensitivity can be achieved over the entire field of view. A happy spatial medium exists for fields several arcminutes in radius; the known cloud has a radius on the order of $1 \mathrm{pc}\left(24^{\prime \prime} .3\right.$ for a distance of $\left.8.5 \mathrm{kpc}\right)$ and a field several arcminutes $\left(1^{\prime} \approx 2.5 \mathrm{pc}\right)$ on a side could accommodate many such clouds. For the velocity coverage, while constrained by the correlator for a given observation, we must appeal to the $l-v$ maps of the Bally et al. (1987) data and the theoretical models we wish to test (Binney et al. 1991). An inspection of the ${ }^{12} \mathrm{CO}(1-0)$ data suggests that the easiest way to view a sizable segment of the postshock region at once is to observe near one of the vertical segments of the shock lanes as projected in the $l-v$ plane thanks to our large range in $\Delta v$ compared to $\Delta l$ or $\Delta b$. We also wish to avoid contamination from line-of-sight molecular emission and larger scale structures of the inner Galaxy. For this study, we mapped a region near the Great Annihilator described by $r \sim 5^{\prime}$ and $\Delta v_{\mathrm{LSR}} \sim 100 \mathrm{~km} \mathrm{~s}^{-1}$ which meets these criteria.

In Section 2, we discuss in detail our observations of the cloud and its environs with both the Combined Array for Research in Millimeter-Wave Astronomy (CARMA) and the James Clerk Maxwell Telescope (JCMT), in Sections 3 and 4 our results and analysis, and in Section 5 the implications of our results, including an assessment of whether our region is "typical."

\section{OBSERVATIONS \& DATA REDUCTION}

\subsection{CARMA D \& E Arrays}

CARMA is a heterogenous interferometric array made up of six 10.4 and nine $6.1 \mathrm{~m}$ radio dishes located at $2195 \mathrm{~m}$ at Cedar Flat in the Inyo Mountains of California. The observatory operates in several different configurations. We report here on two tracks in the compact 2007 August E-Array and one track in the 2008 April D-Array (Table 1) toward the Great Annihilator cloud in $\mathrm{HCN} J=1-0(88.63 \mathrm{GHz})$ and $\mathrm{HCO}^{+}$ $J=1-0(89.19 \mathrm{GHz})$. For these tracks, we made mosaicked maps toward, but slightly offset from, the peak of emission in the Phillips \& Lazio (1995) $\mathrm{HCO}^{+}(1-0)$ map at 17:40:43.0 $-29: 43: 25.0$ (1950.0). The $\mathrm{HCN}$ and $\mathrm{HCO}^{+}$observations were conducted simultaneously with both lines in the upper sideband and with a velocity range from -90 to $-190 \mathrm{~km} \mathrm{~s}^{-1}$. For one of the E-array tracks (baselines 8-66 m) and the D-Array track (baselines 8-108 m), the weather was excellent, whereas the second E-Array track produced usable data requiring substantial 
Table 1

CARMA Observational Parameters

\begin{tabular}{|c|c|c|c|c|c|c|c|c|c|}
\hline Array Config. & $\begin{array}{l}\text { Source Int. Time } \\
(\mathrm{hr})\end{array}$ & \# Mosaic Points & Gain Cal. & Passband Cal. & Flux Cal. & $\begin{array}{l}\mathrm{HCO}^{+} \text {Beam } \\
(\operatorname{arcsec})\end{array}$ & $\begin{array}{c}\mathrm{HCO}^{+} \mathrm{PA} \\
(\text { deg. })\end{array}$ & $\begin{array}{l}\text { HCN Beam } \\
(\operatorname{arcsec})\end{array}$ & $\begin{array}{l}\text { HCN PA } \\
\text { (deg.) }\end{array}$ \\
\hline D08A & 2.36 & 37 & $1733-130$ & $2148+069$ & MWC349 & $15.4 \pm 0.5 \times 6.8 \pm 0.2$ & $1 \pm 2$ & $16.3 \pm 0.6 \times 7.0 \pm 0.2$ & $1 \pm 2$ \\
\hline E07 & 4.43 & 7 & $1733-130$ & $1751+096$ & MWC349 & $27 \pm 1 \times 12.8 \pm 0.5$ & $5 \pm 2$ & $29 \pm 2 \times 13.4 \pm 0.6$ & $3 \pm 2$ \\
\hline
\end{tabular}

Note. Beam sizes and position angles determined using the MIRIAD mospsf task within the central $20^{\prime \prime} \times 20^{\prime \prime}$ of the combined data sets.

flagging. Visibilities in the E-array which had one antenna shadowing another were discarded. While the shortest baselines tracing the largest structures are lost in this procedure, the E-Array data considered by itself nonetheless reproduces the D-Array detections where the fields overlap. Importantly, the E-array includes numerous shorter, nonshadowed baselines significantly different from those in the D-Array, producing significantly improved $u-v$ coverage. To calibrate and analyze the data, we employed the MIRIAD software package (Sault et al. 1995). We customized a standard script provided by CARMA to inspect the data, apply calibrations and flags, and extract clean maps. We used an SDI CLEAN algorithm to generate the maps presented here; a check against a maximum entropy deconvolution scheme showed no significant differences.

Figures 1 and 2 show the integrated amplitude maps for the combined calibrated D \& E-Array data, and it is immediately obvious that there is a large number of features, some of which are spatially resolved, and that many of these features are weak or absent in the $\mathrm{HCO}^{+}$data. The clouds are dense and the spatial coverage of our $r \sim 3^{\prime}$ field between -90 and -190 $\mathrm{km} \mathrm{s}^{-1}$ is about $25 \%$. At a glance, it is clear that we see a large number of features which may have small filling factors in larger beams or survey grids. We clearly detect the Great Annihilator cloud (labeled cloud 1) to the left of map center. Note that because there were fewer E-Array mosaic pointings than in

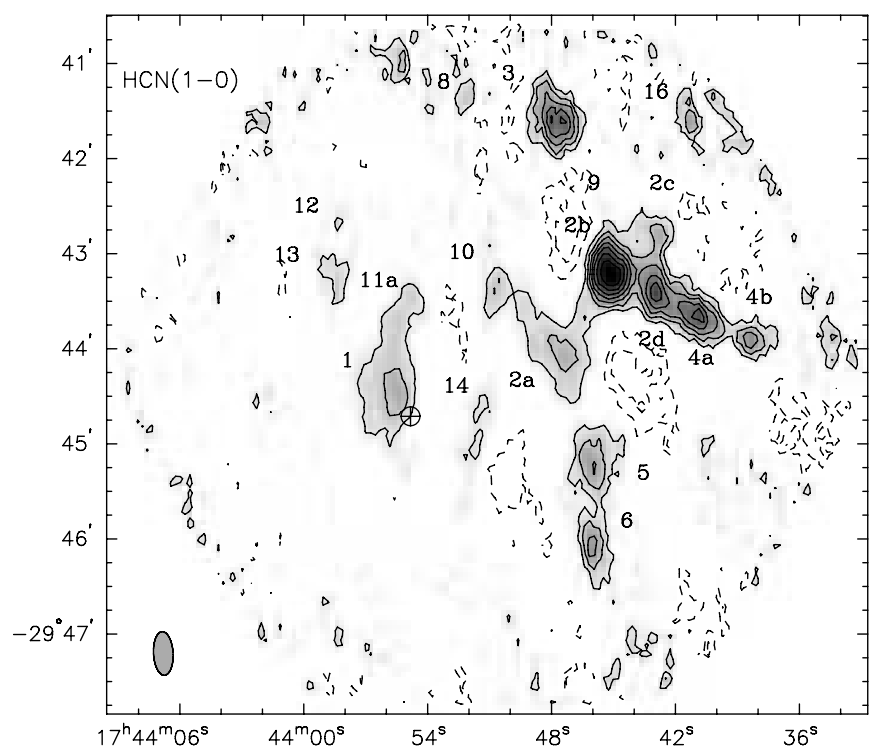

Figure 1. Contour map of integrated amplitude of $\mathrm{HCN}(1-0)$ for combined $\mathrm{D}$ and E-Array data. Dashed contours indicate negatives in the map. The mean rms for the integrated map is $2.6 \mathrm{Jy}_{\text {beam }}^{-1} \mathrm{~km} \mathrm{~s}^{-1}$; a one-contour feature is roughly a $2 \sigma$ detection. Some real detections appear weaker in the map as a result of spatial coincidence with negative features, others are behind brighter clouds (see the text). Clumps in Table 2 have been labeled. A crosshair near cloud 1 shows the location of 1E 1740.7-2942 (Mirabel et al. 1991), and the beam size is at the bottom left. The Galactic equator is not in our field, but is to the top right.
D-Array (Table 1), the signal-to-noise ratio decreases toward the map edge. Because the map contains negative amplitudes, and features at different velocities may overlap spatially, the integrated maps do not accurately reflect the strength of emission in any one cloud; we found clumps that have no labels in Figures 1 and 2 . These clumps are evident in the channel maps (Figures 5 and 6).

\subsection{James Clerk Maxwell Telescope}

We obtained ${ }^{12} \mathrm{CO} J=6-5$ data for the map in Figure 3 on 1995 April 23 with the 15 m JCMT located at the Mauna Kea Observatory. We used the FANATIC submillimeter spectrometer (Harris et al. 1994), a quasi-optical receiver with a solid-state LO and an SIS mixer. For this observing run the mixer device and associated dual-slot antenna was supplied by $\mathrm{J}$. Zmuidzinas; the receiver temperature was about $400 \mathrm{~K}$ (DSB). The weather was excellent, with $55 \%$ zenith transmission at the line's $691 \mathrm{GHz}$ frequency, deduced from measurements of the sky's emission temperature. The intensity scale for Figure 3 is $T_{A}^{*}$ for a forward coupling to a Jupiter-size $\left(\sim 40^{\prime \prime}\right)$ source. Further information on observing and calibration methods may be found in Harris et al. (1995).

The central field of Figure 3 is pointed toward the peak of $\mathrm{HCO}^{+}(1-0)$ emission in Phillips \& Lazio (1995), and the remainder of the spectra are taken from fields offset by $20^{\prime \prime}$ in

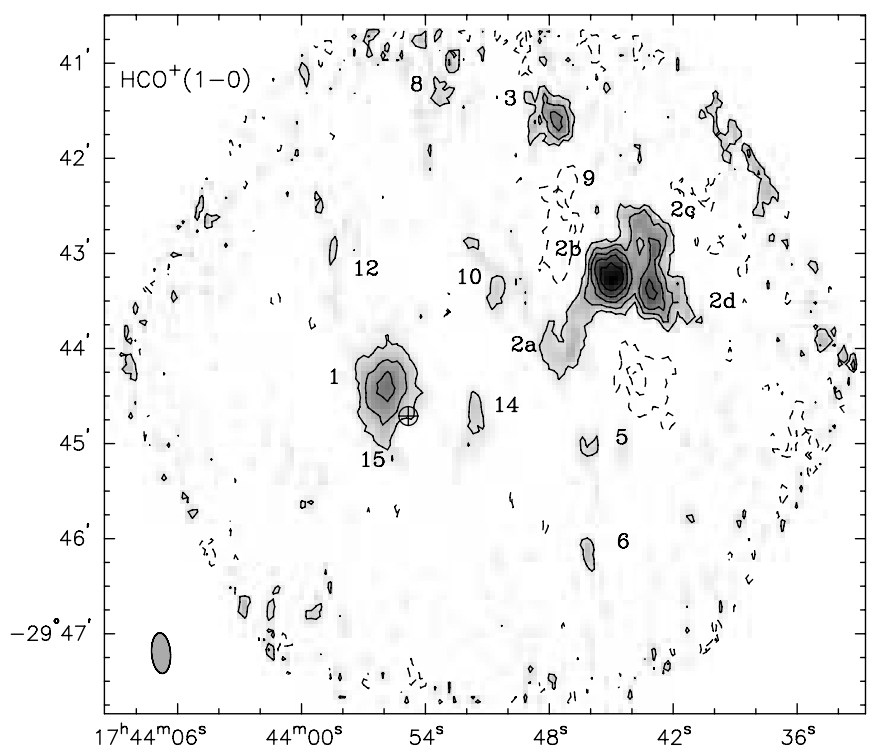

Figure 2. Contour map of integrated amplitude of $\mathrm{HCO}^{+}(1-0)$ for combined $\mathrm{D}$ and E-Array data. Dashed contours indicate negatives in the map. The mean rms for the integrated map is $1.7 \mathrm{Jy}_{\text {beam }}^{-1} \mathrm{~km} \mathrm{~s}^{-1}$; a one-contour feature is roughly a $2 \sigma$ detection. Some real detections appear weaker in the map as a result of spatial coincidence with negative features, others are behind brighter clouds (see the text). Clumps in Table 2 have been labeled. A crosshair near cloud 1 shows the location of 1E 1740.7-2942 (Mirabel et al. 1991), and the beam size is at the bottom left. The Galactic equator is not in our field, but is to the top right. 


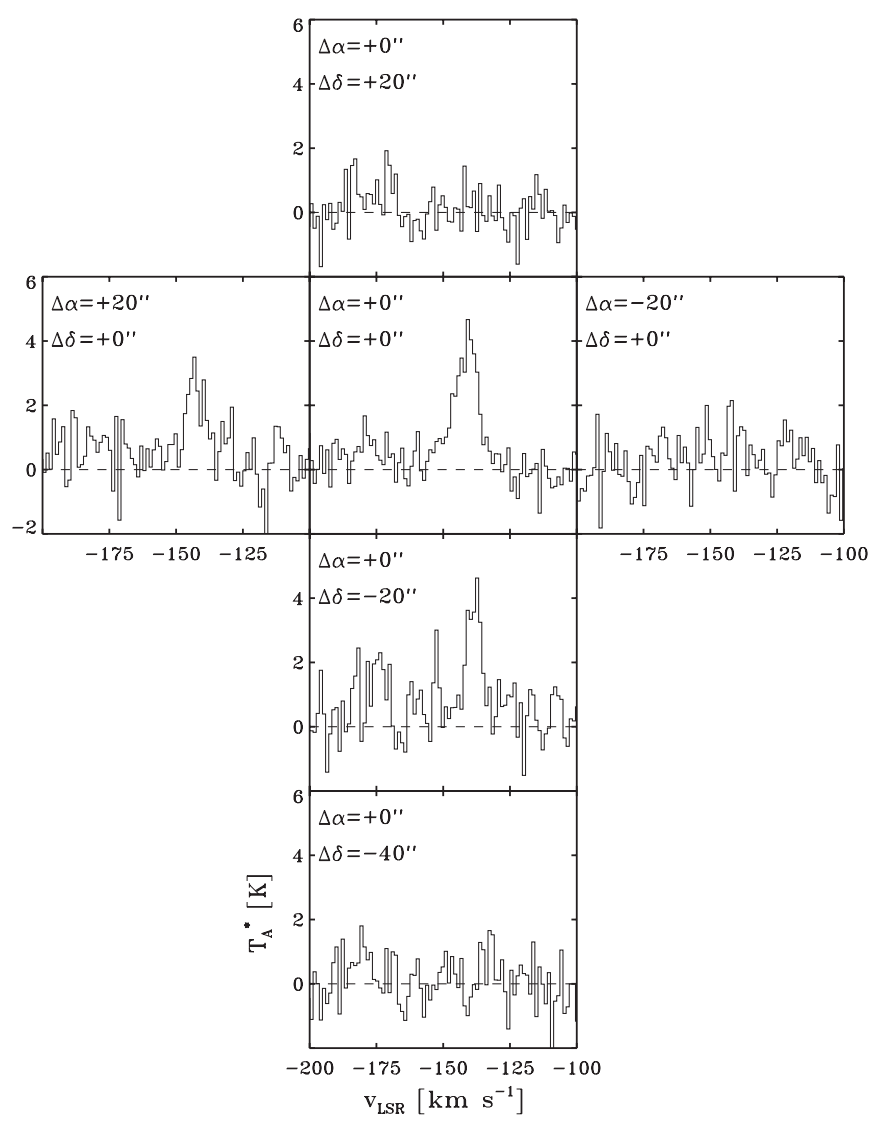

Figure 3. JCMT ${ }^{12} \mathrm{CO} J=6-5$ map toward peak of Phillips \& Lazio (1995) on cloud 1 near the Great Annihilator.

$\alpha$ and $\delta$; each pointing lasted for $240 \mathrm{~s}$. Additional data were obtained in ${ }^{13} \mathrm{CO}(6-5)$ toward the Phillips \& Lazio peak, but no line was detected with an upper limit 20 times smaller than the ${ }^{12} \mathrm{CO}(6-5)$ line brightness toward the same position.

The detection of the cloud in the $J=6-5$ line demonstrates that the cloud is both warm and dense. The approximate size and shape of the cloud traced by Figure 3 is in agreement with the CARMA maps and the Mirabel et al. (1991) $\mathrm{HCO}^{+}(1-0)$ map.

\section{OBSERVED PROPERTIES}

The presence of a number of bright regions in Figures 1 and 2 and the suggestion that these regions may be quite excited is an important result. To measure the properties of these regions, we used the clumpfind algorithm (Williams et al. 1994) using the average rms noise per spectral channel within $3^{\prime}$ of map center and requiring 60 pixels per clump (about twice the area). Clumps found at $r>3^{\prime}$ reside in the low-signal map edge, and were therefore rejected, although we found additional structure in the map edge we do not further discuss here. The clumpf ind results are given in Table 2 and are consistent with a visual inspection in that the same regions are identified. Because clumpfind treats pixels along the $v_{\mathrm{LSR}}$-axis no differently than the spatial pixels, many of the clumps reported are spatially unresolved at the $2 \sigma$ level, and in extracting spectra for these cases we use the beam size instead of the reported clumpfind radii. In Figure 4 we show the spectra extracted for these clumps; for convenience, where clumpfind found multiple clumps which appear to be associated along all three axes we have extracted one average spectrum for the region using the MIRIAD imspect task. These
Table 2

CARMA Measured $\mathrm{HCN} / \mathrm{HCO}^{+}$Clump Properties

\begin{tabular}{|c|c|c|c|c|c|c|c|}
\hline Label & Line & $\begin{array}{c}\alpha \\
(\mathrm{J} 2000) \\
\end{array}$ & $\begin{array}{c}\delta \\
(\mathrm{J} 2000) \\
\end{array}$ & $\begin{array}{c}v_{\mathrm{LSR}} \\
\left(\mathrm{km} \mathrm{s}^{-1}\right)\end{array}$ & $\begin{array}{c}T_{\text {peak }} \\
(\mathrm{K}) \\
\end{array}$ & $\begin{array}{l}R_{\text {clump }} \\
\text { (arcsec) }\end{array}$ & $\begin{array}{l}\Delta v_{\text {FWHM }} \\
\left(\mathrm{km} \mathrm{s}^{-1}\right)\end{array}$ \\
\hline 1 & $\mathrm{HCO}^{+}$ & 174356.1 & -294419.0 & -141.7 & 2.47 & 26.9 & 4.8 \\
\hline 1 & $\mathrm{HCN}$ & 174355.8 & -294422.0 & -145.0 & 1.61 & & 4.1 \\
\hline $2 \mathrm{a}$ & $\mathrm{HCO}^{+}$ & 174348.9 & -294325.0 & -105.6 & 1.90 & & 9.5 \\
\hline $2 \mathrm{a}$ & $\mathrm{HCN}$ & 174348.2 & -294355.0 & -107.0 & 1.43 & 5.0 & 10.6 \\
\hline $2 b^{a}$ & $\mathrm{HCO}^{+}$ & 174344.8 & -294316.0 & -103.9 & 2.51 & 26.9 & 4.8 \\
\hline $2 b$ & $\mathrm{HCN}$ & 174344.8 & -294313.0 & -102.0 & 2.58 & 4.2 & 7.2 \\
\hline $2 b$ & $\mathrm{HCN}$ & 174344.8 & -294313.0 & -107.0 & 2.08 & 20.5 & 5.2 \\
\hline $2 c^{a}$ & $\mathrm{HCO}^{+}$ & 174344.8 & -294316.0 & -103.9 & 2.51 & 26.9 & 4.8 \\
\hline $2 c$ & $\mathrm{HCN}$ & 174342.7 & -294258.0 & -92.1 & 1.24 & 23.0 & 11.6 \\
\hline $2 d$ & $\mathrm{HCO}^{+}$ & 174343.2 & -294325.0 & -105.6 & 1.90 & 25.4 & 9.5 \\
\hline $2 d$ & $\mathrm{HCN}$ & 174342.9 & -294322.0 & -102.0 & 2.31 & 23.5 & 11.8 \\
\hline 3 & $\mathrm{HCO}^{+}$ & 174347.5 & -294137.9 & -117.1 & 1.95 & & 6.3 \\
\hline 3 & $\mathrm{HCN}$ & 174348.0 & -294134.0 & -116.9 & 2.27 & & 9.7 \\
\hline 3 & $\mathrm{HCN}$ & 174347.5 & -294125.0 & -111.9 & 1.89 & 18. & 8.8 \\
\hline $4 a$ & $\mathrm{HCN}$ & 174340.9 & -294340.0 & -123.5 & 1.53 & 1 & 11.0 \\
\hline $4 a$ & $\mathrm{HCN}$ & 174341.1 & -294 & -128.4 & 1.20 & 1 & 6.5 \\
\hline $4 b$ & $\mathrm{HCN}$ & 174338.3 & -294 & -121.8 & 1.40 & 1 & 5.2 \\
\hline $4 \mathrm{~b}$ & $\mathrm{HCN}$ & 174338.3 & -294 & -13 & 1.33 & 5.8 & 8.1 \\
\hline $4 \mathrm{~b}$ & $\mathrm{HCN}$ & 1743 & -294 & -12 & 1.30 & 1 & 3.6 \\
\hline 5 & $\mathrm{HCN}$ & 174345.9 & -294510.0 & -178.0 & 1.24 & 2 & 9.6 \\
\hline 6 & $\mathrm{HCO}^{+}$ & 174345.7 & -294616.0 & -125.3 & 0.92 & 9.7 & 5.9 \\
\hline 6 & $\mathrm{HCN}$ & 174345.9 & -294613.0 & -126.8 & 1.28 & 0 & 5.8 \\
\hline 6 & $\mathrm{HCN}$ & 174345.9 & -294607.0 & -12 & 1.03 & 1 & 2.7 \\
\hline 7 & $\mathrm{HCN}$ & 174350.5 & -294204.0 & -186.3 & 1.50 & 1 & 3.0 \\
\hline 0 & $\mathrm{HCN}$ & 174352.1 & -294119.0 & -10 & 1.48 & & 13.8 \\
\hline 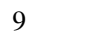 & $\mathrm{HCO}^{+}$ & 174346.2 & -294228.0 & -123.6 & 1.41 & 12.9 & 3.4 \\
\hline 9 & $\mathrm{HCN}$ & 174345.7 & -294228.0 & -120.2 & 1.29 & 1 & 5.1 \\
\hline 10 & $\mathrm{HCO}^{+}$ & 1743 & -294313.0 & -1 & 1.01 & 11.3 & 2.1 \\
\hline 10 & $\mathrm{HCN}$ & 1743 & -294 & -1 & 1.11 & 1 & 3.5 \\
\hline $11 \mathrm{a}$ & $\mathrm{HCN}$ & 174355.6 & -2943 & -182.9 & 1.06 & 13.1 & 3.7 \\
\hline $11 \mathrm{~b}$ & $\mathrm{HCN}$ & 174355.1 & -2944 & -184.6 & 0.85 & 16.3 & 7.6 \\
\hline 12 & $\mathrm{HCO}^{+}$ & 174357.9 & -294307.0 & -126.9 & 1.22 & 14.8 & 1.8 \\
\hline 12 & $\mathrm{HCN}$ & 174358.1 & -294313.0 & -123.49 & 0.87 & 13.0 & 5.6 \\
\hline 13 & $\mathrm{HCN}$ & 174357.7 & -294310.0 & -168.1 & 0.99 & 10.4 & 4.3 \\
\hline 14 & $\mathrm{HCN}$ & 174350.8 & -294434.0 & -115.2 & 0.80 & 11.7 & 3.1 \\
\hline 15 & $\mathrm{HCO}^{+}$ & 174356.3 & -294458.0 & -130.2 & 1.05 & 17.1 & 3.2 \\
\hline $16^{\mathrm{b}}$ & $\mathrm{HCN}$ & 174340.2 & -294128.0 & -105.3 & 2.17 & 11.1 & 4.1 \\
\hline $16^{\mathrm{b}}$ & $\mathrm{HCN}$ & 174341.1 & -294128.0 & -107.0 & 1.82 & 12.3 & 4.9 \\
\hline $16^{\mathrm{b}}$ & $\mathrm{HCN}$ & 174341.6 & -294119.0 & -111.9 & 1.38 & 9.9 & 7.1 \\
\hline $16^{\mathrm{b}}$ & $\mathrm{HCN}$ & 174341.3 & -294122.0 & -97.1 & 1.30 & 9.1 & 6.0 \\
\hline $16^{\mathrm{b}}$ & $\mathrm{HCN}$ & 174341.3 & -294137.0 & -115.2 & 1.20 & 16.3 & 10.5 \\
\hline 17 & $\mathrm{HCN}$ & 174345.2 & -294301.0 & -181.3 & 1.33 & 16.1 & 6.8 \\
\hline 17 & $\mathrm{HCN}$ & 174345.2 & -294255.0 & -188.0 & 1.26 & 9.9 & 4.4 \\
\hline 17 & $\mathrm{HCN}$ & 174345.2 & -294258.0 & -191.2 & 1.23 & 13.7 & 2.7 \\
\hline 18 & $\mathrm{HCN}$ & 174350.1 & -294213.0 & -97.1 & 0.93 & 12.5 & 3.3 \\
\hline 18 & $\mathrm{HCN}$ & 174349.8 & -294225.0 & -88.8 & 0.90 & 10.3 & 3.3 \\
\hline 19 & $\mathrm{HCN}$ & 174351.0 & -294331.0 & -115.2 & 1.11 & 13.0 & 3.5 \\
\hline
\end{tabular}

Notes. clumpf ind results for our data cube. Clumps 1-6 we consider "resolved associations" or "clouds" whereas 7-19 are "unresolved clumps" based on the $2 \sigma$ contours in the integrated map. All features are found within $3^{\prime}$ of the map center.

${ }^{a}$ The $\mathrm{HCO}^{+}$clumpf ind run connected $2 \mathrm{~b}$ and $2 \mathrm{c}$, whereas the $\mathrm{HCN}$ did not.

${ }^{\mathrm{b}}$ Clump 16 was halfway outside our criterion for rejection in the map edge; the rms error on the peak flux is greater than the other clumps, hence more clumps were found with the algorithm.

spectra were extracted from roughly the $2 \sigma$ contour tracing the association boundaries. Using clumpfind introduced a systematic bias into the sizes of the clumps along the spatial and $v_{\mathrm{LSR}}$-axis, and we discuss the implications of this bias on our derived masses in Section 4.1. 


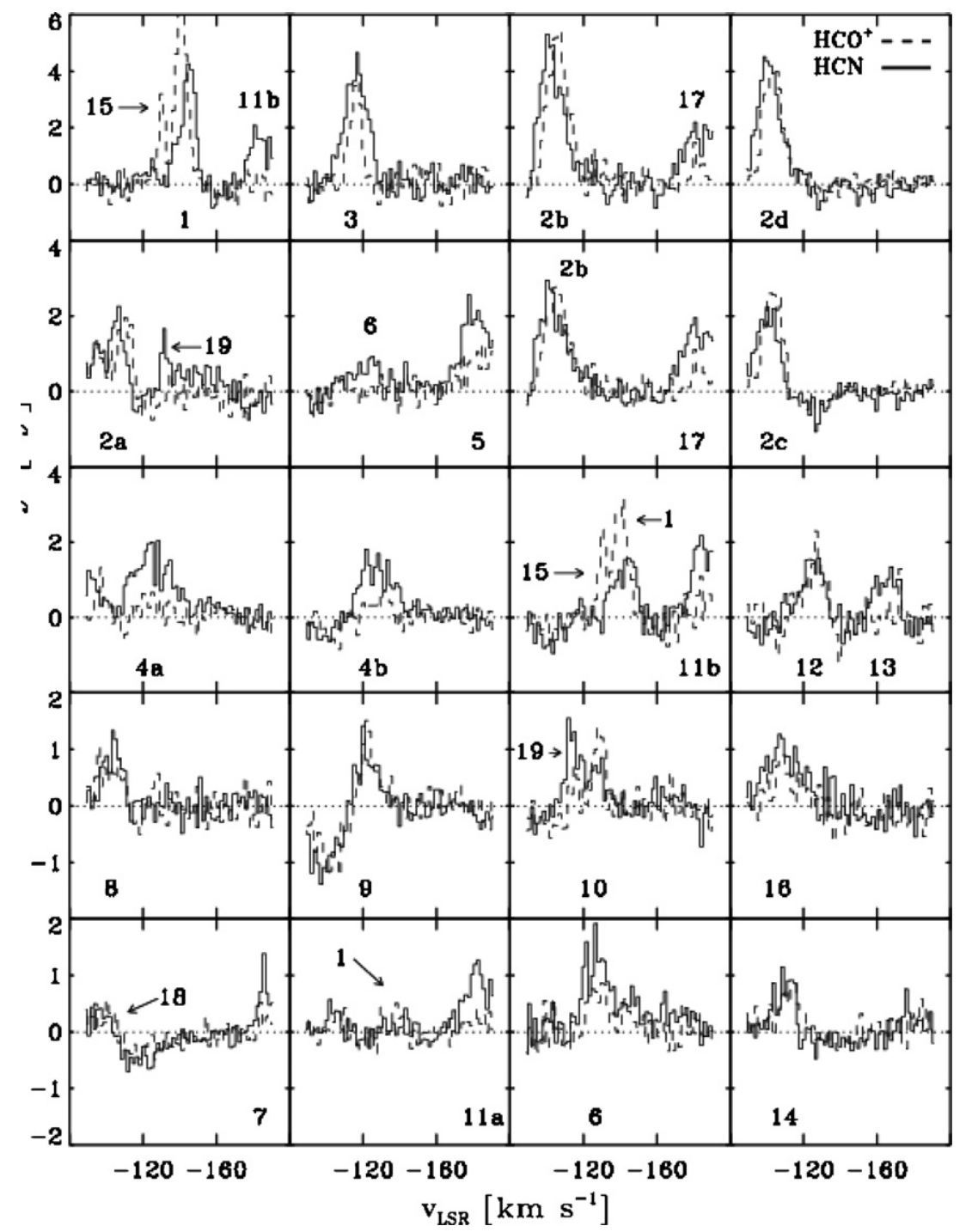

Figure 4. Spectra extracted for clumps and clouds listed in Table 2 from rectangular boxes roughly corresponding to the clump size at the $2 \sigma$ contour. The spectra were extracted from the combined D and E-Array data in each line. The label at the bottom of each frame marks the clump or cloud for which the spectrum was extracted; the same feature in a different frame has lower signal. In cases where the $2 \sigma$ contour was smaller than a beam size, the spectrum was extracted from a rectangle with the height and width of the major and minor axes of the synthesized beam.

\subsection{Average Description}

For our analysis, we divide our clumps into resolved associations and unresolved individual clumps, where the former are identified as groups of clumps in the same region of $\alpha, \delta$, and $v_{\text {LSR }}$. The resolved clumps have characteristic scales of $r \sim 1$ pc $(24$ ". 3 at a distance of $8.5 \mathrm{kpc})$ and, in some cases, several resolved regions may themselves be associated; we consider cloud 2 to be a molecular cloud containing multiple dense regions since we see nearby negative dips at similar peak velocities. Hereafter we adopt "cloud" to refer to resolved associations of clumps found with clumpfind and "clump" to refer to any individual component or unresolved feature. The resolved clouds are labeled 1, 2, 3, 4, 5, and 6, and these are the brightest features in the map; the fainter clouds are unresolved, but may have extended structure we would see with a deeper integration. In the case of clump $2 b$, the algorithm found two clumps in the $\mathrm{HCN}$ data which are spatially coincident and differ by $5 \mathrm{~km}$ $\mathrm{s}^{-1}$ along the $v_{\mathrm{LSR}}$-axis, but a detailed inspection of the channel maps (Figures 5 and 6) convince us that clumpfind ought not to have found two clumps. Similarly, two clumps were found in cloud 3 where a visual inspection suggests that they should be considered one structure. The clumps are listed as the algorithm found them in Table 2, and we use the results from the algorithm through the rest of the analysis, but we note that there may be systematic inaccuracies with our approach. All other clumps found by clumpfind passed visual inspection.

The $\mathrm{HCN}$ and $\mathrm{HCO}^{+}$line strength is comparable in about half of the clumps; perhaps interestingly, only cloud 1 has a significantly stronger $\mathrm{HCO}^{+}$line. On average, where $\mathrm{HCO}^{+}$ emission is present, the FWHM line widths are comparable to the HCN despite differing line strengths. The lines are roughly symmetric, but we lack the signal to rigorously investigate the line profiles. Measured FWHM line widths for individual clumps range from $3 \mathrm{~km} \mathrm{~s}^{-1}$ to $14 \mathrm{~km} \mathrm{~s}^{-1}$ whereas average line widths for the clouds as presented in Figure 4 range from $8 \mathrm{~km}$ $\mathrm{s}^{-1}$ to $30 \mathrm{~km} \mathrm{~s}^{-1}$, assuming the line is adequately represented by a single Gaussian component.

As expected, many of the clumps and associations are seen within the faint emission described in Section 1.2 (Figure 7). 


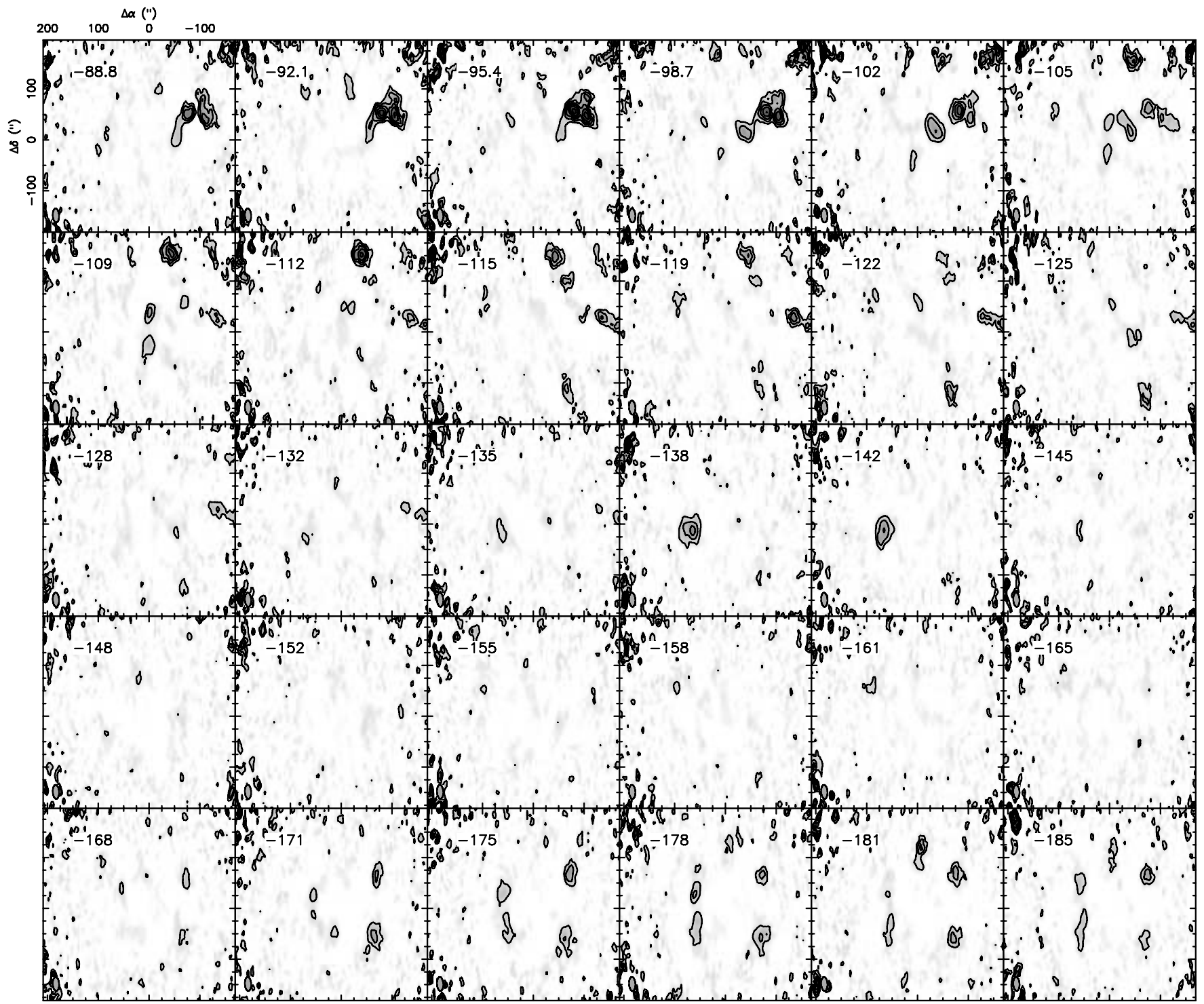

Figure 5. Velocity channel maps for the combined D and E-Array HCN $(1-0)$ data. The 63 original channels have been binned into 30 channels here.

Clump 1, associated with 1E 1740.7-2942, is closer to the $x_{2}$ orbits and may not be part of the same structure. Another group of clumps lies near $-180 \mathrm{~km} \mathrm{~s}^{-1}$ and may lie on the $x_{2}$ orbits, but the grouping in $l$ and $b$ suggests that these clumps are actually on noncircular orbits, since they are grouped between $-0.10<b<-0.08$ and there is a relative lack of emission where the GCR intersects our region in the Bally et al. (1987) data. The apparent clustering in Figure 7 is largely a result of plotting all the clumps found with clumpfind and, when associations such as cloud 2 are considered as one object at some average $(l, v)$, the clumps appear randomly distributed (in latitude, they are distributed preferentially toward the Galactic equator, and we do not have data for $b>-0.03$ ). If the clouds were on $x_{2}$ orbits, we would expect them to be clustered toward larger negative $v_{\mathrm{LSR}}$ near $-180 \mathrm{~km} \mathrm{~s}^{-1}$ at $l \approx 0.9$. The isolated clumps and associations of gas are isolated from one another along all three axes such that it seems unlikely that they are part of one larger complex. This implies that the "bridges" seen in $l-v$ slices of the Bally et al. (1987) data are not coherent structures, but rather, the coherent structures exist on smaller scales. We remark on the individual clouds below.

\subsection{Resolved Clouds}

1. $-\mathbf{1 4 0} \mathrm{km} \mathrm{s}^{-\mathbf{1}}$. Cloud 1 is the molecular cloud associated with $1 \mathrm{E} 1740.7-2942$ and is the only cloud in the sample for which the $\mathrm{HCO}^{+}$line is much stronger than the $\mathrm{HCN}$ line. In the CARMA maps, we do not see the extended structure to the south which Phillips \& Lazio (1995) attribute to different ionization regimes near the jets of 1E 1740.7-2942, but we do see some low-signal emission to the southeast (clump 15) at about $-130 \mathrm{~km} \mathrm{~s}^{-1}$ which may be the "ridge" they describe. The JCMT map does not extend far enough to assess the presence of clump 15 in $\mathrm{CO}$ $J=6-5$, but clearly shows that the highly excited $\mathrm{CO}$ traces the $\mathrm{HCO}^{+}$well in the region observed and that the cloud is compact. Clumps $11 \mathrm{a}$ and $11 \mathrm{~b}$ at $-180 \mathrm{~km} \mathrm{~s}^{-1}$ are spatially coincident with cloud 1 , and may be physically associated with each other. In Section 5.2 we discuss how these results bear on a possible physical association with 1E 1740.7-2942.

2. $-\mathbf{1 0 0} \mathbf{k m ~ s}^{-\mathbf{1}}$. The clumps labeled $2 \mathrm{a}-2 \mathrm{~d}$ are each resolved individually, but are close on the sky, have nearly identical peak velocities, and are near strong negatives in the 


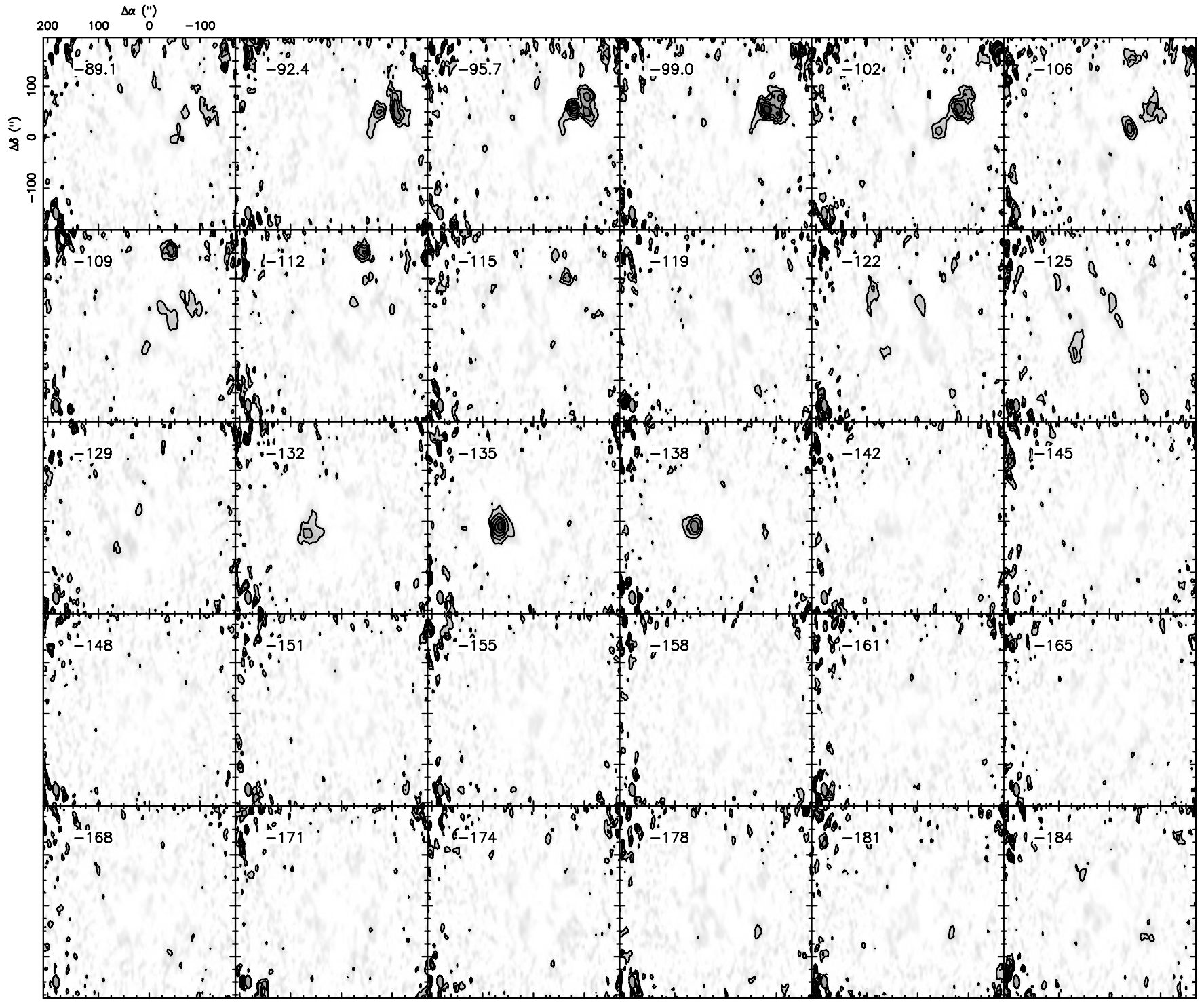

Figure 6. Velocity channel maps for the combined D and E-Array $\mathrm{HCO}^{+}(1-0)$ data. The 63 original channels have been binned into 30 channels here.

deconvolved maps which also appear at the same velocity. These negatives indicate large-scale structure resolved out by the interferometer and motivate a physical picture of several dense clumps within a diffuse envelope. The HCN and $\mathrm{HCO}^{+}$line strengths are roughly equal in each cloud, and $2 \mathrm{~b}$ has the brightest peak flux in the map (Figure 4 shows the $\mathrm{HCO}^{+}$in cloud 1 as stronger because it is summed over an extraction box). However, cloud $2 \mathrm{~b}$ coincides on the sky with a smaller clump at $-180 \mathrm{~km} \mathrm{~s}^{-1}$ which makes the integrated amplitude in the region in Figures 1 and 2 significantly brighter than the contribution from $2 \mathrm{~b}$ alone. Since the $\mathrm{HCO}^{+}$emission is weaker than the $\mathrm{HCN}, 2 \mathrm{c}$ is not identified as a clump in the $\mathrm{HCO}^{+}$data and is rather split into two tails extending from $2 \mathrm{~b}$ and $2 \mathrm{~d}$. We assume that because it is identified as a separate clump in HCN that it ought to be identified separately from the two brighter emission cores.

3. $-\mathbf{1 1 5} \mathbf{k m ~} \mathbf{s}^{-\mathbf{1}}$. This cloud is curious, since the average FWHM line width over the association is significantly smaller in $\mathrm{HCO}^{+}$than $\mathrm{HCN}$ despite the strong emission in both lines. This effect is noticeable in the individual clumps found in the region, and is pronounced even when the spectrum is extracted from a box within contours greater than $4 \sigma$, indicating that perhaps the $\mathrm{HCO}^{+}$in the cloud is confined to the densest regions.

4. $-\mathbf{1 2 5} \mathbf{~ k m ~ s}^{-1}$. Clouds $4 \mathrm{a}$ and $4 \mathrm{~b}$ have similar peak velocities and line widths in their average spectra, and both are much weaker in the $\mathrm{HCO}^{+}$line than the $\mathrm{HCN}$. The clouds are notable for their broad lines which clumpfind breaks up into strings of smaller clumps at similar velocities. The similar peak velocities mean that $4 \mathrm{a}$ and $4 \mathrm{~b}$ are physically separated by less than $1^{\prime}$ and suggest they are or were part of the same complex, although we do not see negative amplitudes that would point to a diffuse envelope. Cloud 6 is similar in line width, size, and line strength to either component of cloud 4, but it is several arcminutes away.

5. $-\mathbf{1 8 0} \mathbf{k m ~ s}^{-1}$. This cloud is close on the sky to cloud 6 and is similarly weak in $\mathrm{HCO}^{+}$, so the extracted spectrum includes a small portion of cloud 6 which appears as a weak feature at $-125 \mathrm{~km} \mathrm{~s}^{-1}$. Cloud 5 is the only certainly resolved cloud at $-180 \mathrm{~km} \mathrm{~s}^{-1}$, although clump 17 behind cloud $2 \mathrm{~b}$ may barely be resolved. 


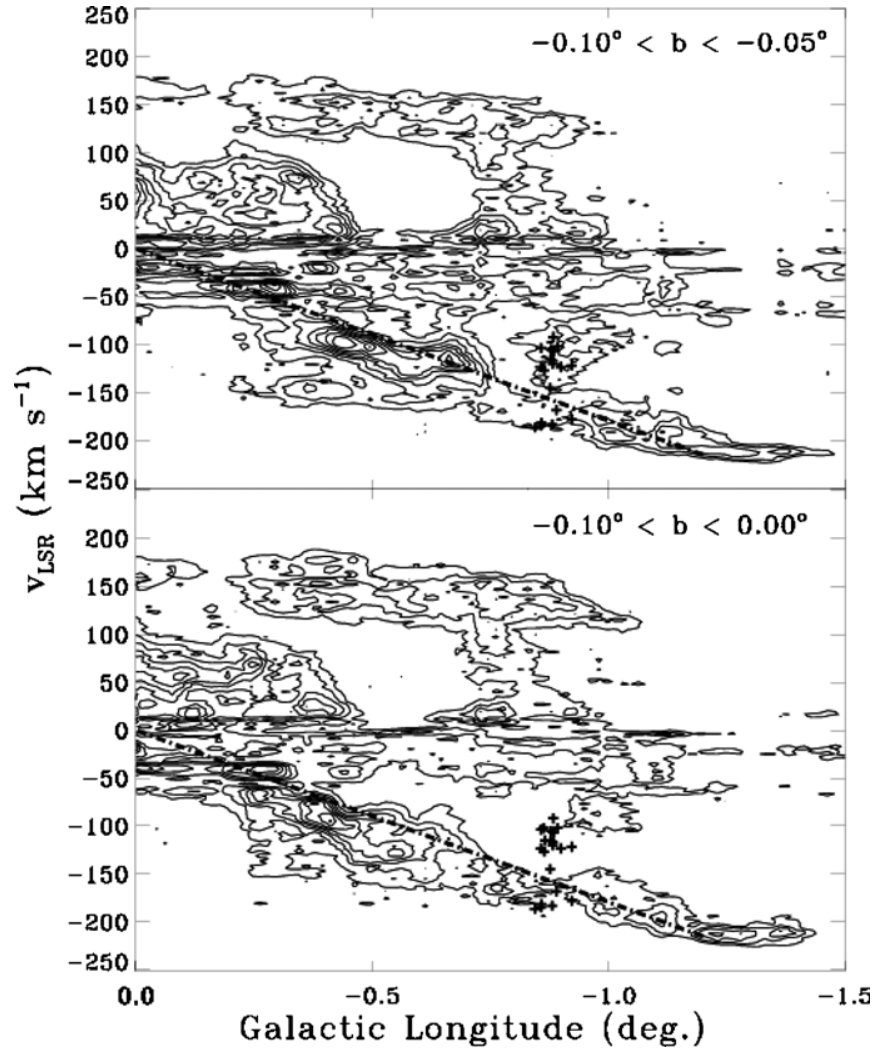

Figure 7. Bally et al. (1987) ${ }^{12} \mathrm{CO}(1-0) l-v$ maps for (top) $-0.10<b<$ -0.05 and (bottom) $-0.10<b<0.00$ with crosses denoting the coordinates of peak emission for our clumps as determined by clumpfind. The diagonal dashed line indicates the approximate location of the $x_{2}$ orbits at this latitude range. Contours range from $2 \mathrm{~K}$ to $30 \mathrm{~K}$ in intervals of $\Delta T=2 \mathrm{~K}$.

Table 3

CARMA HCN Derived Properties

\begin{tabular}{|c|c|c|c|c|c|}
\hline Label & $\begin{array}{l}\Delta v_{\text {FWHM }} \\
\left(\mathrm{km} \mathrm{s}^{-1}\right) \\
\end{array}$ & $\begin{array}{c}R \\
(\mathrm{pc}) \\
\end{array}$ & $\begin{array}{c}M_{\mathrm{vir}} \\
\left(10^{4} M_{\odot}\right) \\
\end{array}$ & $\begin{array}{l}\log _{10}\left[\bar{n}\left(\mathrm{H}_{2}\right)\right] \\
\log \left[\left(\mathrm{cm}^{-3}\right)\right]\end{array}$ & $\begin{array}{c}t_{\text {cross }} \\
\left(10^{5} \mathrm{yr}\right) \\
\end{array}$ \\
\hline 1 & 4.1 & 0.99 & 0.39 & 4.43 & 2.37 \\
\hline $2 a$ & 10.6 & 1.03 & 2.67 & 5.23 & 0.95 \\
\hline $2 b$ & 7.2 & 1.00 & 1.19 & 4.93 & 1.36 \\
\hline $2 b$ & 5.2 & 0.84 & 0.53 & 4.79 & 1.59 \\
\hline $2 c$ & 11.6 & 0.95 & 2.95 & 5.38 & 0.80 \\
\hline $2 d$ & 11.8 & 0.97 & 3.12 & 5.38 & 0.80 \\
\hline 3 & 9.7 & 0.91 & 1.98 & 5.26 & 0.92 \\
\hline 3 & 8.8 & 0.78 & 1.39 & 5.31 & 0.87 \\
\hline $4 a$ & 11. & 0.71 & 1.99 & 5.59 & 0.63 \\
\hline $4 a$ & 6.5 & 0.60 & 0.59 & 5.28 & 0.91 \\
\hline $4 \mathrm{~b}$ & 5.2 & 0.67 & 0.42 & 4.98 & 1.27 \\
\hline $4 \mathrm{~b}$ & 8.1 & 0.65 & 0.99 & 5.40 & 0.79 \\
\hline $4 b$ & 3.6 & 0.65 & 0.19 & 4.70 & 1.76 \\
\hline 5 & 9.6 & 0.82 & 1.76 & 5.34 & 0.84 \\
\hline 6 & 5.8 & 0.77 & 0.60 & 4.97 & 1.29 \\
\hline 6 & 2.7 & 0.51 & 0.09 & 4.65 & 1.87 \\
\hline
\end{tabular}

Notes. The clump radius in pc uses a galactocentric distance of $8.5 \mathrm{kpc}$. When multiple clumps were detected in one resolved association, all clumps were included even if they were individually unresolved; we only calculate masses for resolved clouds.

\subsection{Unresolved Clumps}

As mentioned above, several clumps listed in Table 2 appear in the channel maps and not in the integrated amplitude maps. This occurs either when the clump coincides with another cloud on the sky (as in the case of clump 17 behind $2 \mathrm{~b}$ ) or with a negative at a different $v_{\mathrm{LSR}}$ (as in the case of clump 9). The "unresolved" clumps do not appear to be a distinct population from the resolved clouds, and are instead characterized by weaker emission. We extracted average spectra from regions of about a beam size; clumpfind does find smaller spatial sizes for these clumps than for the ones comprising resolved associations. The line widths of the unresolved clumps are in agreement with those of the resolved ones.

\section{DERIVED PROPERTIES}

We have detected $\sim 20$ clumps of gas in a $3^{\prime}$ radius field. These clumps have $r \leqslant 1 \mathrm{pc}$ (assuming a distance of $8.5 \mathrm{kpc}$ ) and may reside in clouds with radii of a few parsecs. The clumps have line widths, fluxes, positions, and peak velocities that make it difficult to characterize them as different parts of the same structure. Integrating across $\Delta v=100 \mathrm{~km} \mathrm{~s}^{-1}$, the clumps cover about $25 \%$ of our field. The gas tends to emit more strongly in $\mathrm{HCN}$ than $\mathrm{HCO}^{+}$, and at least one of these clouds (cloud 1) produces a ${ }^{12} \mathrm{CO}(6-5)$ line which traces the $\mathrm{HCO}^{+}$and $\mathrm{HCN}$ gas quite well, meaning the cloud is highly excited. Surveys in ${ }^{12} \mathrm{CO}(4-3)$ emission show that clouds near our field are excited, although we know from comparing the JCMT ${ }^{12} \mathrm{CO}$ $(6-5)$ and unpublished ${ }^{12} \mathrm{CO}(2-1)$ data that not all gas in the region are so highly excited.

We now use these measured properties to derive additional quantities for our clouds, and on the basis of these results, propose that the sources are dense $\left(n_{\mathrm{H}_{2}}>10^{5} \mathrm{~cm}^{-3}\right)$, and that they have shocked recently. Because of the weakness of the $\mathrm{HCO}^{+}$in many of the clumps, our arguments regarding the dense gas rely on the $\mathrm{HCN}$ data.

\subsection{Mass \& Density Estimates}

Mass estimates invariably reflect their underlying physical assumptions, so further testing of these assumptions is required to assess their accuracy. The virial mass in particular is very uncertain for Galactic center clumps. Although the external pressures appear to be an order of magnitude too low to bind clumps on our scales (Miyazaki \& Tsuboi 2000), the clumps may be bound by the strong magnetic fields known to exist in the Galactic center, or may be unbound. The virial mass is primarily useful as a way to compare our results to other work in the absence of better estimators but adopting values from previous work is fraught with peril, since the structure observed is necessarily dependent on the beam size.

The sensitivity of the virial mass to line width means that whether we take $M_{\mathrm{vir}}=\Sigma_{i} m_{\mathrm{vir}, i}$, where a cloud is made up of clumps with mass $m_{\mathrm{Vir}, \mathrm{i}}$, or $M_{\mathrm{vir}}=\bar{\sigma}_{v}^{2} R / G$, where $\bar{\sigma}_{v}$ is taken from an average description such as Figure 4, will influence our results. The line width-size relation (Larson 1981; Miyazaki \& Tsuboi 2000) cannot be used to resolve such ambiguity because in our case it is systematic. Reported line widths also generally appear to reflect the beam size used. We investigated the extent to which noise influences clumpfind in order to determine whether multiple clumps found in resolved clouds accurately describe substructure. There is almost certainly substructure present: the question is whether clumpfind accurately detects it. A visual inspection of the channel maps in addition to testing clumpfind's behavior with artificial resolved clouds in a featureless corner of our map convinced us that the two clumps found in both $2 b$ and 3 in the $\mathrm{HCN}$ are not real, and that a single clump is a better description. On the 
other hand, using the line width from the average spectra in Figure 4 likely overestimates the mass more severely than clumpfind underestimates it. We therefore report the clumpfind results and posit that the masses and densities contained in the resolved clouds are somewhat higher. Table 3 contains the results for each individual clumpf ind detection, $m_{\mathrm{vir}, i}$. In determining $\bar{n}_{\mathrm{H}_{2}}$ for each clump, we used $\mu=1.4 m_{\mathrm{H}}$.

Is using the virial mass reasonable? We know the clouds must be dense, since typical critical densities for HCN $(1-0)$ are $n \geqslant$ $10^{5} \mathrm{~cm}^{-3}$, so the assumption that they are gravitationally bound is not wholly unreasonable, although a shock compression might induce similar densities which would then dissipate. The line widths indicate macroscopic turbulent support and are roughly symmetric, so if the clouds are bound, they are unlikely to be much more concentrated than at virialized relaxation. We posit that for dense, noncollapsing gas the virial mass is an adequate order of magnitude description, but note that other mass estimates from studies with larger beam sizes disagree with the virial mass by up to an order of magnitude (Miyazaki \& Tsuboi 2000).

In the resolved clouds our derived densities range from $10^{4.4}$ to $10^{5.6} \mathrm{~cm}^{-3}$ and, as we stated, we believe our results underestimate the virial mass. If the LTE mass is a better descriptor of the "real" mass (Miyazaki \& Tsuboi 2000), then our densities are $\sim 10$ times too high and, therefore, typical for Galactic center clumps with radii 2-10 times larger than those of our clumps. If our clumps are smaller structures inside larger diffuse clouds such as those resolved with larger beams, then they must either be much more dense than $10^{4} \mathrm{~cm}^{-3}$, or else comprise most of the mass and volume of their hosts; for clumps $\sim 10$ times larger in radius the latter seems unlikely. The convergence of the critical density and size arguments lends some credence to the virial estimates as an order of magnitude estimate, so we believe our resolved clouds contain large amounts of gas at densities exceeding $10^{5} \mathrm{~cm}^{-3}$. The brightnesses and line widths of the unresolved clumps suggest that they contain gas at similar densities, so we estimate that the total mass contained in our cube is $(1-3) \times 10^{5} M_{\odot}$, contained in parsec-scale bundles with a small filling factor. We note that since the $\mathrm{HCO}^{+}$line widths generally disagree with the $\mathrm{HCN}$ in a given cloud, the $\mathrm{HCO}^{+}$virial masses would be different, but the $\mathrm{HCO}^{+}$lines have a lower SNR, making the measurements more uncertain.

\subsection{Crossing Time}

Independently of the virial mass, we know the density to be quite high from the critical density required to excite $\mathrm{HCN}$ $(1-0)$. In an inspection of Two Micron All Sky Survey $J$-, $H$-, and $K$-band images of our field, we do not see enhanced star formation activity associated with our resolved clouds; the clouds have not yet collapsed, so they are either unbound and will decompress in a crossing time or bound and close to virialized. In either case, the clumps must be quite young if they are starless, since they appear to have turbulent support. The crossing time, $t_{\text {cross }} \sim R / \Delta v_{\text {FWHM }}$, is about $10^{5} \mathrm{yr}$ (Table 3 ) for our resolved clouds. Assuming a circular orbit at $180 \mathrm{pc}$ from the Galactic center with a velocity of $200 \mathrm{~km} \mathrm{~s}^{-1}$ (the highest LSR velocities seen in the CO parallelogram Binney et al. 1991), the orbital period is $\sim 5 \times 10^{6} \mathrm{yr}$, over an order of magnitude greater than the dynamical timescale. It is likely that the clumps have recently fallen onto their present orbits, so they probably experienced a recent shock. However, the constituents of cloud 2 suggest that condensation and collapse may occur.

\section{DISCUSSION}

\subsection{The Postshock Voids}

The Binney et al. (1991) hypothesis identifying the CO parallelogram with the ILR of the Galactic bar may not be correct in detail (Ferrière et al. 2007), but the identification of the CO parallelogram with starless dust lane shock zones (RodriguezFernandez et al. 2006) strengthens the proposed mechanism for molecule formation in shocks near the ILR. The large surface density of molecular gas in the Galactic center and orbit families in the barred potential make it all but certain that continuous inflow from the $\mathrm{H}$ I disk occurs.

The density, excitation, and $(l, v)$ distribution of our clumps suggests that we are indeed seeing molecular gas which has recently formed in these shock zones. Owing to their density, the clumps represent a significant amount of mass (greater than $10^{5} M_{\odot}$ in our field); this mass is in a region where the orbits guarantee eventual transport to the $x_{2}$ disk. This mass is transported in small bundles, possibly as a spray of material from streamer collisions. If these bundles are unbound and decompress, diffuse molecular gas may rain down onto the GCR, otherwise they may experience ballistic impacts with the diffuse molecular envelopes in the GCR. The small filling factor of the dense regions may also explain the voids seen in larger surveys, although, as noted in Section 1.2, larger-scale diffuse emission was detected in the Bally et al. (1987) maps in the region. We expect that regions of similar size in the CMZ where faint emission is hidden by integration across latitude slices should contain gas similar to what we see. Since the gas must be on self-intersecting orbits, the voids may also imply a characteristic timescale for clumps to fall to the $x_{2}$ disk.

There are two challenges to the proposal outlined above. First, the clumps and clouds may be associated by a larger, bridge-like structure. This would imply a directed mass flow at certain points on the $\mathrm{CO}$ parallelogram rather than a large number of small bundles of shocked gas distributed throughout the region in between the shock lanes and the $x_{2}$ orbits. Second, the multiple constituents of cloud 2 in a larger envelope (but still small on the scales of large surveys) suggest that condensation into dense clumps, as seen in Galactic molecular clouds, may take place under higher pressures. The first case is difficult to assess because a close inspection of individual latitude slices of the Bally et al. (1987) ${ }^{12} \mathrm{CO}(1-0)$ data shows many small features in between the CO parallelogram of Binney et al. (1991) and the GCR. We consider it unlikely that these represent steadystate channels for mass flow because of the nature of the selfintersecting $x_{1}$ orbits interior to the ILR. However, it is possible that material from molecular and atomic gas clouds shredded in the shock lanes maintains some coherent structure as it dissipates angular momentum. The second challenge may fit neatly into the first if larger structures fall onto the self-intersecting $x_{1}$ orbits, but aside from the constituents of cloud 2, we see little evidence for dense bridges between otherwise distinct clumps. Furthermore, we see no evidence for star formation, so even internal turbulent shocks must have been recent. If a mechanism for recent shocks is required, the proximity to the shock lanes provides a natural explanation.

To determine whether the Great Annihilator region appears typical, we applied the scale of our map $\left(r \sim 5^{\prime}, \Delta v_{\mathrm{LSR}}=100\right.$ $\mathrm{km} \mathrm{s}^{-1}$ ) to other areas along the shock lanes labeled in Rodriguez-Fernandez et al. (2006) using the Bally et al. (1987) data cube. For the diagonal portions of the $\mathrm{CO}$ parallelogram, we stretched our scale in longitude and shrunk it in $\Delta v$. Ignoring 
the regions near $v_{\mathrm{LSR}}=0 \mathrm{~km} \mathrm{~s}^{-1}$ and obvious large-scale structures such as the GCR, we find that the 1E 1740.7-2942 region for our observation is typical of the postshock regions in the ${ }^{12} \mathrm{CO}(1-0)$ map. The emission associated with this region in the Bally et al. (1987) data appears to come from a structure covering $\Delta b \sim 0.05, \Delta l \sim 0.15$, and $\Delta v \sim 100 \mathrm{~km} \mathrm{~s}^{-1}$. We think it unlikely that the structure is bridged to the $x_{2}$ orbits based on a detailed inspection of the region at different emission contour intervals. We therefore have no reason to believe that the presence of the Great Annihilator in the region detracts from a gas-dynamical analysis. However, we note that this analysis assumes that the observed similarities between regions in the Bally et al. (1987) ${ }^{12} \mathrm{CO}(1-0)$ maps correspond to physical similarities in the environments of the shock lanes. Although the region is small, our results are largely consistent with the Binney et al. (1991) theory of molecule-forming shocks at the ILR even though, in detail, the scenario is more complex. If we are seeing clumps that have recently shocked, it is harder to fit them into alternative pictures explaining the CO parallelogram, e.g., an "expanding molecular ring" or the Stark et al. (2004) proposal of a stalled ring of gas accumulating from the true ILR farther away from the Galactic center.

We know that the giant molecular clouds on the $x_{2}$ disk are forming stars from cores in clumps, but we do not know whether the clumps primarily form via condensation or external perturbation, nor the filling factor of small clumps in gas accreting onto these orbits. Jenkins \& Binney (1994) found in their sticky-particle hydrodynamical models that the steadystate $x_{2}$ disk contains a large number of strongly shocked clumps of gas, although the decompression timescale suggests that if the clouds are still dense, they must be at least gravitationally bound if they experience no additional shocks interior to the dust lanes. Their simulations also find that although there are places where shocked gas preferentially joins the $x_{2}$ disk (e.g., the $l=1.3$ near-side molecular complex), bundles of gas fall in from the shock zone at the ILR at many angles. Jenkins \& Binney (1994) admit that their results are only marginally successful at reproducing the features of the Galactic center, and subsequent models (e.g., Rodriguez-Fernandez \& Combes 2008) have done much better. Yet the results we present here suggest that at least the accretion of material onto the $x_{2}$ disk in the form of dense bundles is still a possibility.

\subsection{IE $1740.7-2942$}

Our data provide useful contrasts to two of the arguments made previously for a physical association between $1 \mathrm{E}$ 1740.7-2942 and cloud 1, but we cannot rule out a possible association. The argument in favor of physical association due to a small chance of coincidental superposition of a black hole with a bright molecular cloud depends in detail on choosing a cutoff brightness. The 5\%-7\% chance previously reported (Bally \& Leventhal 1991; Mirabel et al. 1991) relied on studying the density of peaks in molecular line surveys near the Galactic center using some brightness criterion. Without establishing a similar criterion, we cannot directly compute the probability of a chance association. However, the presence of a large number of clumps in the region with similar line widths and a wide range of peak fluxes suggests that a highly excited clump is common enough not to require association with a black hole. At approximately the $2 \sigma$ contour, the chance of coincidental superposition with any clump in our field is $25 \%$. That the $\mathrm{HCO}^{+}$is noticeably stronger in cloud 1 is unusual, but there are smaller clumps where $\mathrm{HCO}^{+}$is stronger than $\mathrm{HCN}$. With a larger veloc- ity range, the chance of coincidental superposition at arbitrary velocity may well increase.

The Phillips \& Lazio (1995) argument in favor of physical proximity relies on the interpretation of a ridge of $\mathrm{HCO}^{+}$emission parallel to the VLA jets associated with 1E 1740.7-2942 as evidence for an ionization rate gradient. Their ridge is significant (greater than $2 \sigma$ ) and separated from the VLA jets by $15^{\prime \prime}$ with a peak velocity close to $-140 \mathrm{~km} \mathrm{~s}^{-1}$, suggesting that it is part of the same structure as cloud 1, shown in Figure 2 in Phillips \& Lazio (1995). The primary detection of cloud 1 in their OVRO map agrees well with both our CARMA and JCMT data in spatial extent, but we do not detect the southern ridge apparent in their map. Instead, we see clump 15 at $-130 \mathrm{~km}$ $\mathrm{s}^{-1}$ to the southeast of cloud $1-\mathrm{it}$ is closer to the VLA jets than $15^{\prime \prime}$. An inspection of clump 15 in $(l, v)$ space shows a clear separation between it and cloud 1. Moreover, this clump is detected in the JCMT ${ }^{12} \mathrm{CO}(2-1)$ data (not shown) whereas we do not see it in ${ }^{12} \mathrm{CO}(6-5)$. Mirabel et al. (1991) also detect the clump in ${ }^{12} \mathrm{CO}(2-1)$ and not in $\mathrm{CS}(2-1)$. There is undoubtedly emission to the south of cloud 1 , but it is not as excited or warm as cloud 1, nor do we see evidence for a "ridge" linking it to cloud 1 . These results call into question whether an ionization rate gradient exists. Furthermore, Lepp \& Dalgarno (1996) argue, based on their model, that if the $\mathrm{HCO}^{+}$ ridge is caused by an ionization rate gradient, we would expect to see $\mathrm{HCN}$ molecules surviving closer to the black hole. There is no evidence in our maps that $\mathrm{HCN}$ emission associated with cloud 1 is closer to the Chandra X-ray source than the $\mathrm{HCO}^{+}$. Our results make it more difficult to make the case for physical proximity between the Great Annihilator and cloud 1.

\section{CONCLUSIONS}

We have used CARMA D \& E-Array observations in tandem with JCMT data to study the region near 1E 1740.7-2942, a relative "void" in $(l, v)$ diagrams of ${ }^{12} \mathrm{CO}$ survey data. The most important result of our work is the discovery that even in the regions with low average emissivity, there is a substantial amount of mass (greater than $10^{5} M_{\odot}$ ) contained in small, dense, excited regions implying a recent shock. These bundles have scales of $r \sim 1 \mathrm{pc}$ and appear randomly distributed in $(l, v)$ space, so shocks or sprays in the nearby dust lanes of the CO parallelogram naturally explain our observations. The small filling factor of the dense bundles accounts for why they are not seen or only faintly present in large surveys. We investigate in detail the relationship between cloud 1 and the LMXB 1E 1740.7-2942 and argue that the probability of coincidental superposition with excited gas is higher than originally estimates, and that we see no evidence for a proposed ionization rate gradient; we can explain why cloud 1 is excited in the JCMT map without invoking a black hole.

This research was funded in part by the Astronomy Department of the University of Maryland at College Park. The authors thank M. Leventhal for useful discussion of the diffuse $511 \mathrm{keV}$ annihilation radiation in the Galactic plane, and R. Genzel \& J. Zmuidzinas for motivation and support of the JCMT observations. We used $7 \mathrm{~m}$ Bell Labs data made publically available by J. Bally.

Support for CARMA construction was derived from the states of California, Illinois, and Maryland, the Gordon and Betty Moore Foundation, the Kenneth T. and Eileen L. Norris Foundation, the Associates of the California Institute of Technology, 
and the National Science Foundation. Ongoing CARMA development and operations are supported by the National Science Foundation under a cooperative agreement, and by the CARMA partner universities. This material is based on work supported by the National Science Foundation under grant numbers AST0540450 and AST-0540399.

\section{REFERENCES}

Bally, J., \& Leventhal, M. 1991, Nature, 353, 234

Bally, J., Stark, A., Wilson, R., \& Henkel, C. 1987, ApJS, 65, 13

Bania, T. M. 1977, ApJ, 216, 381

Binney, J., Gerhard, O., Stark, A., Bally, J., \& Uchida, K. 1991, MNRAS, 252, 210

Bitran, M., Alvarez, H., Bronfman, L., May, J., \& Thaddeus, P. 1997, A\&AS, 125,99

Ferrière, K., Gillard, W., \& Jean, P. 2007, A\&A, 467, 611

Fux, R. 1999, A\&A, 345, 787

Harris, A. I., Avery, L. W., Schuster, K. F., Tacconi, L. J., \& Genzel, R. 1995, ApJ, 446L, 85

Harris, A. I., Schuster, K. F., Genzel, R., Plathner, B., \& Gundlach, K. H. 1994, Int. J. Infrared Millim. Waves, 15, 1465

Jenkins, A., \& Binney, J. 1994, MNRAS, 270, 703

Larson, R. B. 1981, MNRAS, 194, 809

Lee, C. W. 1996, ApJS, 105, 129

Lepp, S., \& Dalgarno, A. 1996, A\&A, 306, L21
Linke, R., Stark, A, \& Frerking, M. 1981, ApJ, 243, 147

Liszt, H. S., \& Burton, W. B. 1978, ApJ, 225, 815

Main, D. S., Smith, D. M., Heindl, W. A., Swank, J., Leventhal, M., Mirabel, I. F., \& Rodríguez, L. F. 1999, ApJ, 525, 901

Martin, C., Walsh, W., Xiao, K., Lane, A., Walker, C., \& Stark, A. 2004, ApJS, 150,239

Mirabel, I., Morris, M., Wink, J., Paul, J., \& Cordier, B. 1991, A\&A, 251, L43

Miyazaki, A., \& Tsuboi, M. 2000, ApJ, 536, 357

Oka, T., Hasegawa, T., Sato, F., Tsuboi, M., \& Miyazaki, A. 1998, ApJS, 118, 455

Phillips, J., \& Lazio, T. 1995, ApJ, 442, L37

Qin, S., Zhao, J., Moran, J., Marrone, D., Patel, N., Wang, J., Liu, S., \& Kuan, Y. 2008, ApJ, 677, 353

Rodriguez-Fernandez, N. J., \& Combes, F. 2008, A\&A, 489, 115

Rodriguez-Fernandez, N. J., Combes, F., Martin-Pintado, J., Wilson, T. L., \& Apponi, A. 2006, A\&A, 455, 963

Sault, R. J., Teuben, P. J., \& Wright, M. C. H. 1995, in ASP Conf. Ser. 77 , Astronomical Data Analysis Software and Systems IV, ed. R. A. Shaw, H. E. Dayne, \& J. J. E. Hayes (San Francisco, CA: ASP), 433

Stark, A., Bally, J., Knapp, G., \& Wilson, R. 1988, Molecular Clouds in the Milky Way and External Galaxies, ed. R. L. Dickman, R. L. Snell, \& J. S. Young (New York: Springer), 303

Stark, A., Martin, C., Walsh, W., Xiao, K., Lane, A., \& Walker, C. 2004, ApJ, 614, L41

Tsuboi, M., Handa, T., \& Ukita, N. 1999, ApJS, 120, 1

Weidenspointner, G., et al. 2008, Nature, 451, 159

Williams, J., de Geus, E., \& Blitz, L. 1994, ApJ, 428, 693 